\title{
Crystallographic Characterization of Whiskers Using EBSD
}

\author{
J. R. Michael, B. B. McKenzie, and D. F. Susan
}

Materials Science and Engineering Center, Sandia National Laboratory, PO Box 5800, Albuquerque, NM 87185

The crystallographic characterization of micrometer or nanometer-sized whiskers, filaments or rods, which is important to help explain their peculiar shape and unique properties, is challenging. Full crystallographic characterization of whiskers includes the determination of the axis of growth, axis perpendicular to the growth axis and the angle of the whisker with respect to the sample surface normal. A topic of recent research interest is the development of Sn-whiskers on lead-free plating or solders where the whiskers may grow to lengths that may cause failure through shorting of closely spaced electrical conductors. These whiskers have been shown to be single crystals and to grow from grains embedded in the electroplated tin film. [1]

The traditional method of studying the crystallography of whiskers or high-aspect ratio particles in the transmission electron microscopy (TEM) is generally unsatisfactory as the whiskers must be removed from the substrate and only small diameter whiskers can be fully characterized. Electron backscatter diffraction (EBSD) in the scanning electron microscope (SEM) has been shown to be useful in the study of whiskers, nano-rods and nano-wires. [2,3,4,5]

There are many ways that EBSD can be used to characterize whiskers, but the most useful method must characterize the whiskers on the growth substrate. The technique used in this work accomplishes this. First a whisker is aligned with the tilt axis of the SEM and the projected length of the whisker measured. The substrate with the selected whisker is then carefully tilted for EBSD and patterns from the whisker are collected and indexed and the orientation matrix recorded. Simple geometry allows the angle of the whisker with respect to the surface to be measured from SEM images. The whisker growth axis may be precisely determined through mathematical rotation of the measured orientation matrix using the independently measured whisker angle. This method is preferred as it allows the growth axis of the whisker and the surface normal of the grain from which the whisker grew to be measured and the associated growth angle determined independently.

Figure 1 is an example of single crystal Sn-whiskers that have grown out of an electroplated $\mathrm{Sn}$ surface. The whiskers and the substrate patterns can be indexed as the tetragonal form of Sn. Figures $2 \mathrm{a}$ and $\mathrm{b}$ are inverse pole figures of $31 \mathrm{Sn}$-whisker orientations. The growth axis was determined by rotating the orientation matrix through the measured angle of the whisker with respect to the surface normal. Figure 2a shows the crystallographic orientations of the growth axis of the whiskers. It is apparent that the whiskers form with specific growth axes with the most common axes of $<001>$ and $<010>$. Fig. $2 b$ is an inverse pole figure that shows the orientations of the grains that formed whiskers with respect to the surface normal. Each pole is labeled with the growth direction of the corresponding whisker. It is apparent that specific whisker growth directions are not associated with specific grain orientations in the tin plated layer.

Whisker studies performed in this manner preserve more information about the whiskers than typical TEM studies as the whiskers are not removed from the substrate. This method allows a significant 
number of whiskers to be characterized in an efficient manner. Orientations measured with the procedure discussed here will be useful in further understanding of the nucleation and growth of Snwhiskers on electroplated Sn surfaces. This procedure is the most generally applicable EBSD technique for full orientation characterization of whiskers or fibers.

\section{References}

[1] W. J. Boettinger et al., Acta Mat.. 53 (2005) 5033.

[2] B. Hutchinson et al.,Materials Science Forum, 467-470 (2004) 465.

[3] A. Motayed et al., Appl. Phys. Lett., 90 (2007) 043104-1.

[4] J. P. Long, et al., Nano Lett., 7 (2007) 831.

[5] A. Motayed, et al., J. Appl. Phys., 100 (2006) 024306-1.

[6] Sandia National Laboratories is a multi-program laboratory operated by Sandia Corporation, a wholly owned subsidiary of Lockheed Martin Company, for the U.S. Department of Energy's National Nuclear Security Administration under contract DE-AC04-94AL85000.

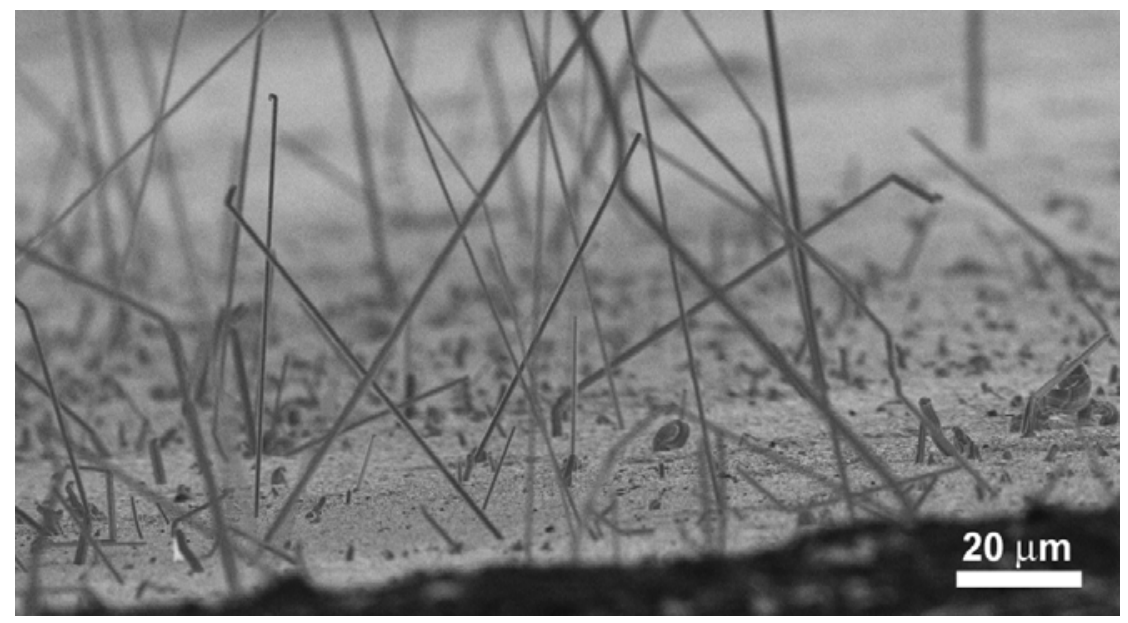

FIG. 1. Sn-whiskers found on electroplated Sn substrates.
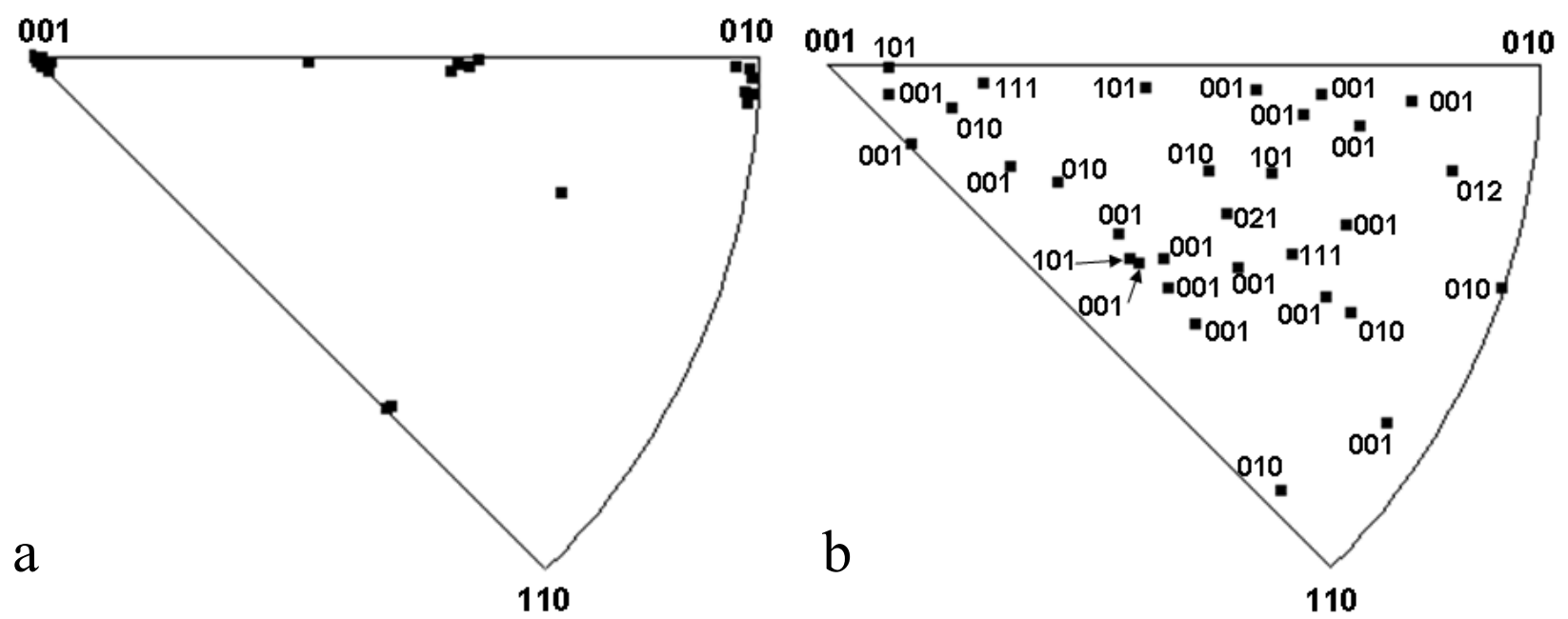

FIG. 2. Inverse pole figures of Sn-whiskers. a) with respect to the growth axis of the whisker b) with respect to the surface normal of the grain at the base of the whisker. Labels represent the growth axis of individual whiskers. 